\title{
Does self-monitoring of blood glucose improve outcome in type 2 diabetes? The Fremantle Diabetes Study
}

\author{
W. A. Davis • D. G. Bruce • T. M. E. Davis
}

Received: 4 October 2006 / Accepted: 6 December 2006 / Published online: 20 January 2007

(C) Springer-Verlag 2007

\begin{abstract}
Aims/hypothesis To assess whether self-monitoring of blood glucose (SMBG) is an independent predictor of improved outcome in a community-based cohort of type 2 diabetic patients.

Materials and methods We used longitudinal data from (1) 1,280 type 2 diabetic participants in the observational Fremantle Diabetes Study (FDS) who reported SMBG and diabetes treatment status at study entry (1993-1996), and (2) a subset of 531 participants who attended six or more annual assessments (referred to as the 5-year cohort). Diabetes-related morbidity, cardiac death and all-cause mortality were ascertained at each assessment, supplemented by linkage to the Western Australian Data Linkage System.

Results At baseline, 70.2\% (898 out of 1,280) of type 2 patients used SMBG. During 12,491 patient-years of follow-up (mean $9.8 \pm 3.5$ years), 486 (38.0\%) type 2 participants died (196 [15.3\%] from cardiac causes). SMBG was significantly less prevalent in those who died during follow-up than in those who were still alive at the end of June 2006 (65.4 vs 73.0\%, $p=0.005$ ). In Cox proportional hazards modelling, after adjustment for confounding and explanatory variables, SMBG was not independently associated with all-cause mortality, but was associated with a 79\% increased risk of cardiovascular mortality in patients not treated with insulin. For the 5-year cohort, timedependent SMBG was independently associated with a $48 \%$ reduced risk of retinopathy.
\end{abstract}

W. A. Davis $(\varangle) \cdot$ D. G. Bruce $\cdot$ T. M. E. Davis School of Medicine and Pharmacology,

University of Western Australia, Fremantle Hospital,

P.O. Box 480, Fremantle, WA 6959, Australia

e-mail:wdavis@cyllene.uwa.edu.au
Conclusions/interpretation SMBG was not independently associated with improved survival. Inconsistent findings relating to the association of SMBG with cardiac death and retinopathy may be due to confounding, incomplete covariate adjustment or chance.

Keywords Cohort study - Epidemiology · Outcomes . Self-monitoring of blood glucose - Type 2 diabetes
Abbreviations
ACR urinary albumin:creatinine ratio
CVD cerebrovascular disease
FDS Fremantle Diabetes Study
HR hazard ratio
ICD International Classification of Diseases
OHA oral hypoglycaemic agent
PAD peripheral arterial disease
ROSSO Retrolective Study 'Self-monitoring of Blood Glucose and Outcome in Patients with Type 2 Diabetes'
SMBG self-monitoring of blood glucose
WADLS Western Australian Data Linkage System

\section{Introduction}

Intensive glycaemic control is a cost-effective way of reducing the complications associated with type 2 diabetes [1]. Whether self-monitoring of blood glucose (SMBG) can improve glycaemia is subject to debate [2, 3]. The Retrolective Study 'Self-monitoring of Blood Glucose and Outcome in Patients with Type 2 Diabetes' (ROSSO) investigators recently reported that SMBG was associated with decreased diabetes-related morbidity and all-cause 
mortality in type 2 diabetes, despite the SMBG group having a higher mean baseline fasting plasma glucose than the non-SMBG group [4]. In an Australian communitybased cohort of type 2 patients we found that SMBG was not a determinant of glycaemic control [5], and now investigate whether, as in ROSSO, those who performed SMBG had better outcomes than those who did not.

\section{Subjects and methods}

Subjects The Fremantle Diabetes Study (FDS) was a longitudinal observational study in a community of 120,097 people in the state of Western Australia. We identified 2,258 subjects between 1993 and 1996, using all available sources, and recruited 1,426 (63\%) to attend annual assessments, of whom 1,294 (91\%) had type 2 diabetes [6]. The FDS protocol was approved by the Fremantle Hospital Human Rights Committee and all subjects gave informed consent. The present study included (1) 1,280 type 2 patients (mean age $64.1 \pm 11.3$ years, $48.8 \%$ men) with complete diabetes treatment and mortality data who reported SMBG status at FDS entry, and (2) a subset of 531 patients (mean age $62.4 \pm 9.4$ years, $54.2 \%$ men) who attended six or more consecutive annual assessments.

Clinical assessment At baseline and annual reviews, a comprehensive history was taken and a physical examination was performed. Complications were identified using standard criteria [5, 7]. Microalbuminuria was defined as an urinary albumin:creatinine ratio $(\mathrm{ACR}) \geq 3.0 \mathrm{mg} / \mathrm{mmol}$ on a first morning sample, neuropathy as a score $>2 / 8$ on the clinical portion of the Michigan Neuropathy Screening Instrument, and retinopathy as any grade in one/both eyes on direct and/or indirect ophthalmoscopy and/or detailed specialist assessment. Self-report and hospitalisations were used to identify cerebrovascular disease (CVD; stroke, transient ischaemic attack) and CHD (myocardial infarction, angina, coronary revascularisation). Peripheral arterial disease (PAD) was defined as an ankle:brachial index $\leq 0.9$ or diabetes-related amputation. Blindness in one or both eyes, foot amputation and end-stage renal disease were also defined to allow comparison with the ROSSO findings [4].

Hospital morbidity and mortality Western Australian government registers record details of all deaths and hospital admissions, and make up part of the Western Australian Data Linkage System (WADLS) [8], which provided endpoint data to the end of June 2006. The Confidentiality of Health Information Committee approved linkage with the FDS database. All hospitalisations for endpoints were identified using the International Classification of Diseases (ICD)-9$\mathrm{CM}$ and ICD-10-AM diagnosis/procedure codes. Causes of death were classified independently by two authors (D.G. Bruce, T.M.E. Davis) as 'cardiac' or 'other' [7].

Statistical analysis Data were analysed using SPSS for Windows (version 11.5) and are presented as proportions or means $\pm \mathrm{SD}$. Comparison of two independent proportions was by Fisher's exact test. Freedom from non-fatal endpoints, all-cause mortality or cardiac death was analysed by baseline SMBG status using the KaplanMeier method and compared with the log-rank test. For outcomes with sufficient events, Cox proportional hazards modelling (forward conditional variable entry and removal with $p<0.05$ and $p>0.05$, respectively), was used to determine: (1) independent predictors of first ever occurrence of endpoints, with all clinically plausible univariate variables with a $p$ value of less than 0.20 considered for entry; and (2) whether, after adjusting for these variables, either SMBG at baseline or the time-dependent covariate SMBG was independently associated with outcome. A $p$ value of less than 0.05 was considered significant.

\section{Results}

At baseline, 898 type 2 patients (70.2\%) performed SMBG. During 12,491 patient-years of follow-up (mean $9.8 \pm 3.5$ years), $486(38.0 \%)$ died, of which $196(15.3 \%)$ deaths were from cardiac causes. SMBG was significantly less prevalent in those who died during follow-up than in those who were alive at the end of June 2006 (65.4 vs $73.0 \%, p=0.005)$.

$S M B G$ and mortality In unadjusted survival analysis (Model 1), SMBG was associated with a significant $24 \%$ reduction in all-cause mortality (Table 1). After adjusting for age, sex and diabetes duration (Model 2), this became a non-significant $11 \%$ increased risk. Additional adjustment for independent risk factors for all-cause mortality (Model 3 ) did not alter this finding. In patients on diet \pm oral hypoglycaemic agents (OHAs) and in those on insulin, baseline SMBG was associated with significant unadjusted $24 \%$ and $54 \%$ reductions in the risk of death, respectively, which became non-significant after full adjustment.

In unadjusted models for cardiac death, there was a significant $55 \%$ risk reduction in insulin-treated patients (Table 1); after full adjustment, this became non-significant. SMBG was associated with a $55 \%$ increased risk of cardiac death in Model 3 as a result of a significant $79 \%$ increased risk in patients not treated with insulin.

Five-year cohort Consistent with intensification of diabetes therapy, and as reported previously [5], the proportion of 


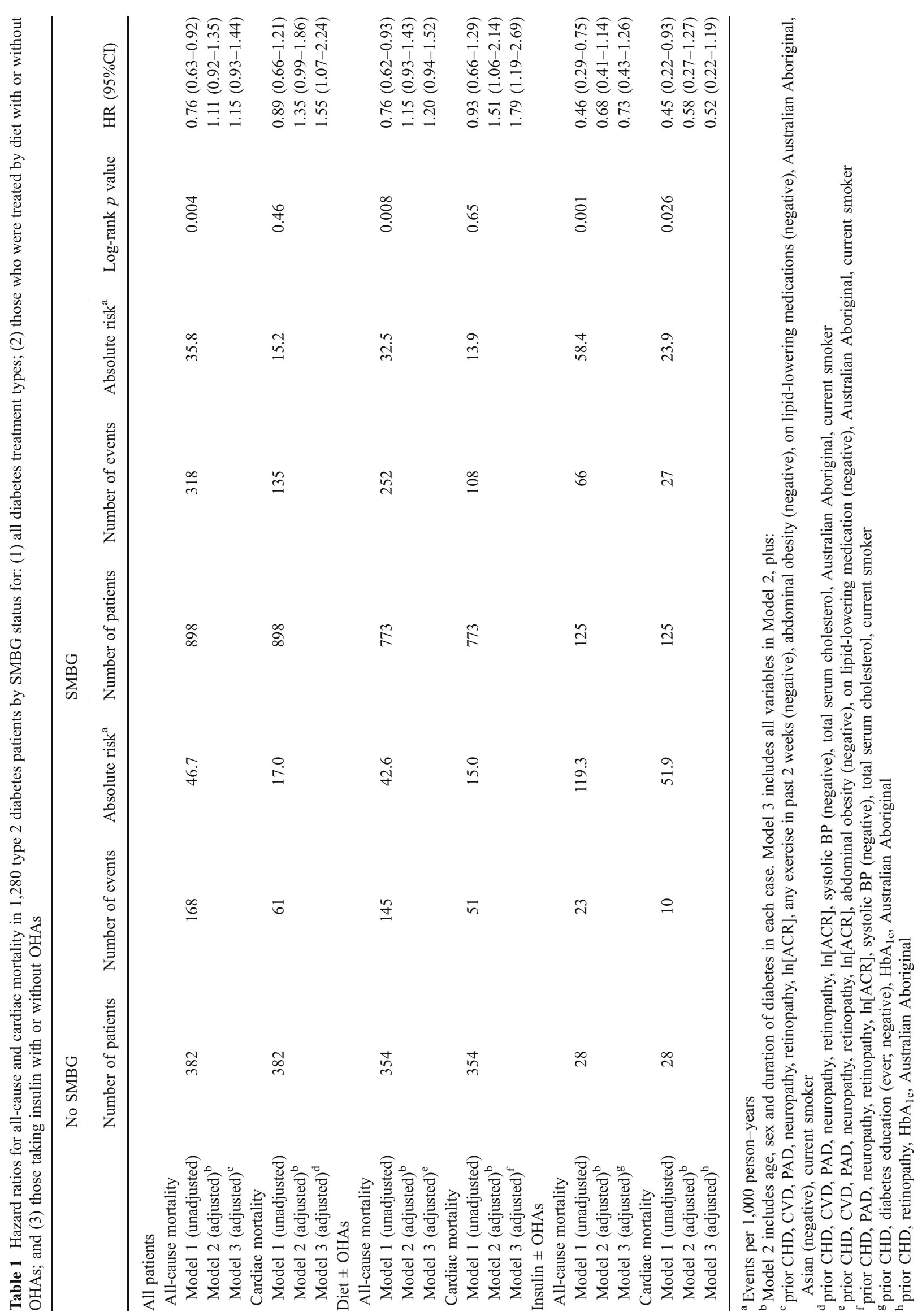


the 5-year longitudinal cohort using SMBG increased over a mean of $5.4 \pm 0.5$ years, from $75.2 \%$ at entry to $85.5 \%$ at third review, with little change thereafter.
We ascertained the incidence of first ever occurrence of micro- and macrovascular complications by baseline SMBG status (Table 2). Unadjusted survival analysis

Table 2 Hazard ratios of first-ever non-fatal complications in the 531 type 2 diabetes patients in the longitudinal arm for single and combined micro- and macrovascular events, and the ROSSO Study non-fatal endpoint, by SMBG status at: (1) baseline; and (2) each annual assessment (time-dependent covariate)

\begin{tabular}{|c|c|c|c|c|c|c|c|c|c|}
\hline & \multicolumn{3}{|c|}{ No SMBG at baseline } & \multicolumn{3}{|c|}{ SMBG at baseline } & \multirow[b]{2}{*}{$\begin{array}{l}\text { Log-rank } \\
p \text { value }\end{array}$} & \multirow[b]{2}{*}{$\operatorname{HR}(95 \% \mathrm{CI})$} & \multirow{2}{*}{$\begin{array}{l}\text { Time-dependent } \\
\text { SMBG use } \\
\text { HR }(95 \% \text { CI })\end{array}$} \\
\hline & $\begin{array}{l}\text { No. of } \\
\text { patients }\end{array}$ & $\begin{array}{l}\text { No. of } \\
\text { events }\end{array}$ & $\begin{array}{l}\text { Absolute } \\
\text { risk }^{\mathrm{a}}\end{array}$ & $\begin{array}{l}\text { No. of } \\
\text { patients }\end{array}$ & $\begin{array}{l}\text { No. of } \\
\text { events }\end{array}$ & $\begin{array}{l}\text { Absolute } \\
\text { risk }^{\mathrm{a}}\end{array}$ & & & \\
\hline \multicolumn{10}{|l|}{ Retinopathy } \\
\hline Model 1 (unadjusted) & 116 & 47 & 90.0 & 349 & 112 & 68.4 & 0.11 & $0.76(0.54-1.07)$ & $0.51(0.36-0.72)$ \\
\hline Model 2 (adjusted) ${ }^{\mathrm{b}}$ & & & & & & & & $0.80(0.57-1.13)$ & $0.52(0.37-0.74)$ \\
\hline Model 3 (adjusted) ${ }^{\mathrm{c}}$ & & & & & & & & $0.82(0.58-1.16)$ & $0.52(0.37-0.73)$ \\
\hline \multicolumn{10}{|l|}{ Neuropathy } \\
\hline Model 1 (unadjusted) & 84 & 62 & 199.3 & 256 & 186 & 203.0 & 0.76 & $1.05(0.78-1.40)$ & $0.77(0.57-1.03)$ \\
\hline Model 2 (adjusted) & & & & & & & & $1.16(0.87-1.55)$ & $0.79(0.59-1.07)$ \\
\hline Model 3 (adjusted) ${ }^{\mathrm{d}}$ & & & & & & & & $1.30(0.97-1.74)$ & $0.89(0.66-1.20)$ \\
\hline \multicolumn{10}{|l|}{ Microalbuminuria } \\
\hline Model 1 (unadjusted) & 82 & 37 & 104.1 & 258 & 103 & 90.6 & 0.38 & $0.85(0.58-1.23)$ & $0.77(0.52-1.14)$ \\
\hline Model 2 (adjusted) ${ }^{\mathrm{b}}$ & & & & & & & & $0.88(0.60-1.28)$ & $0.78(0.53-1.15)$ \\
\hline Model 3 (adjusted) ${ }^{\mathrm{e}}$ & & & & & & & & $0.91(0.62-1.33)$ & $0.74(0.50-1.10)$ \\
\hline \multicolumn{10}{|l|}{ Any microangiopathy } \\
\hline Model 1 (unadjusted) & 58 & 50 & 280.5 & 172 & 153 & 302.6 & 0.59 & $1.09(0.79-1.51)$ & $0.99(0.71-1.38)$ \\
\hline Model 2 (adjusted) $^{\mathrm{b}}$ & & & & & & & & $1.22(0.89-1.68)$ & $0.96(0.69-1.33)$ \\
\hline Model 3 (adjusted) ${ }^{\mathrm{f}}$ & & & & & & & & $1.34(0.97-1.86)$ & $0.98(0.71-1.37)$ \\
\hline \multicolumn{10}{|l|}{ Myocardial infarction } \\
\hline Model 1 (unadjusted) & 131 & 6 & 8.6 & 386 & 18 & 8.8 & 0.97 & $1.02(0.41-2.57)$ & $0.58(0.24-1.41)$ \\
\hline Model 2 (adjusted) ${ }^{\mathrm{b}}$ & & & & & & & & $1.13(0.44-2.89)$ & $0.63(0.26-1.54)$ \\
\hline \multicolumn{10}{|l|}{ Stroke } \\
\hline Model 1 (unadjusted) & 133 & 2 & 2.7 & 398 & 2 & 0.9 & 0.28 & $0.35(0.05-2.52)$ & $0.19(0.03-1.36)$ \\
\hline Model 2 (adjusted) ${ }^{\mathrm{b}}$ & & & & & & & & $0.82(0.10-6.60)$ & $0.30(0.04-2.28)$ \\
\hline \multicolumn{10}{|c|}{ Peripheral arterial disease } \\
\hline Model 1 (unadjusted) & 101 & 51 & 119.3 & 307 & 134 & 99.3 & 0.29 & $0.84(0.61-1.16)$ & $0.74(0.53-1.04)$ \\
\hline Model 2 (adjusted) ${ }^{b}$ & & & & & & & & $0.97(0.70-1.36)$ & $0.80(0.57-1.13)$ \\
\hline Model 3 (adjusted) $^{\mathrm{g}}$ & & & & & & & & $1.13(0.79-1.62)$ & $0.89(0.62-1.28)$ \\
\hline \multicolumn{10}{|l|}{ Any macroangiopathy } \\
\hline Model 1 (unadjusted) & 99 & 54 & 133.7 & 297 & 133 & 103.8 & 0.11 & $0.77(0.56-1.06)$ & $0.67(0.48-0.93)$ \\
\hline Model 2 (adjusted) ${ }^{\mathrm{b}}$ & & & & & & & & $0.88(0.63-1.21)$ & $0.71(0.51-0.99)$ \\
\hline Model 3 (adjusted) ${ }^{\mathrm{h}}$ & & & & & & & & $0.88(0.63-1.22)$ & $0.74(0.52-1.04)$ \\
\hline \multicolumn{10}{|l|}{ All vascular disease } \\
\hline Model 1 (unadjusted) & 44 & 41 & 222.0 & 141 & 128 & 230.1 & 0.95 & $0.99(0.69-1.41)$ & $1.38(0.89-2.15)$ \\
\hline Model 2 (adjusted) & & & & & & & & $1.15(0.80-1.64)$ & $1.43(0.91-2.23)$ \\
\hline Model 3 (adjusted) ${ }^{\mathrm{i}}$ & & & & & & & & - & - \\
\hline \multicolumn{10}{|l|}{ ROSSO endpoint } \\
\hline Model 1 (unadjusted) & 110 & 8 & 13.8 & 328 & 26 & 15.3 & 0.76 & $1.13(0.51-2.50)$ & $0.54(0.26-1.14)$ \\
\hline Model 2 (adjusted) ${ }^{\mathrm{b}}$ & & & & & & & & $1.29(0.58-2.87)$ & $0.58(0.27-1.23)$ \\
\hline
\end{tabular}

${ }^{\mathrm{a}}$ Events per 1,000 person-years

${ }^{\mathrm{b}}$ Model 2 includes age, sex and duration of diabetes in each case. Model 3 includes all variables in Model 2, plus:

${ }^{\mathrm{c}}$ fasting plasma glucose

${ }^{\mathrm{d}}$ Asian (negative), other European, mixed/other ethnicity (negative)

${ }^{\mathrm{e}} \mathrm{BMI}, \ln [\mathrm{ACR}]$, aspirin-use, any exercise in past 2 weeks (negative), married (negative)

${ }^{\mathrm{f}} \ln [\mathrm{ACR}]$

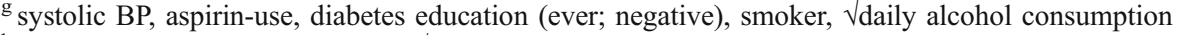

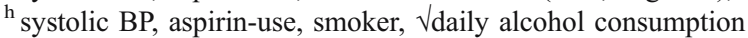

${ }^{\mathrm{i}}$ none after adjustment for ${ }^{\mathrm{b}}$ 
showed no significant benefit of baseline SMBG ( $p \geq$ 0.11 ) for any endpoint, single or combination. Neither adjustment for age, sex and diabetes duration (Model 2), nor relevant additional variables independently associated with the outcomes in Cox proportional hazards modelling (Model 3), improved this result.

Time-dependent Cox models were included to allow for commencement (or discontinuation) of SMBG during follow-up. In both unadjusted and adjusted models, SMBG during follow-up was associated with a reduction in the risk of retinopathy of approximately $50 \%$. In unadjusted analysis, the risk of the development of any macrovascular disease was reduced by $33 \%$ in patients who used SMBG, but this became non-significant after full adjustment.

SMBG was not associated with the first occurrence of the composite ROSSO endpoint (which occurred infrequently), either at baseline or as a time-dependent covariate.

\section{Discussion}

The present study represents the second observational assessment of the relationship between SMBG and outcome in type 2 diabetes. With regard to all-cause mortality in the full FDS sample, our unadjusted data showed that baseline SMBG was associated with a significant risk reduction, a relationship observed in both non-insulin-treated and insulin-treated subgroups. After adjustment for other potential explanatory variables, this apparent benefit was lost in both subgroups. For both combined micro- and macroangiopathy outcomes, SMBG was not associated with a first ever event in the 5-year longitudinal cohort in either unadjusted or adjusted models, with or without timedependent analyses. These findings are inconsistent with those of the ROSSO study [4], in which all-cause mortality and diabetes-related morbidity were reduced by 32 and $51 \%$, respectively, in patients employing SMBG.

What is the explanation for these discrepancies? ROSSO had a larger number of patients than the FDS (3,268 vs $1,280)$, followed for a similar period. However, while the FDS involved a prevalent, prospectively-studied, community-based cohort and all patients had access to subsidised SMBG [5], ROSSO was 'retrolective'-newly-diagnosed patients aged $\geq 45$ years were selected-and only those receiving insulin were reimbursed for SMBG [4]. Whereas there was a low rate of migration of the FDS sample out of Western Australia [9] (96\% had been captured on the WADLS by the end of June 2006 [10]), the retention rate of the ROSSO primary care sample is unknown. The range of potential explanatory variables (including diabetes education, marital status, education, alcohol consumption, exercise and urinary ACR) and the completeness of data collection (e.g. $\mathrm{HbA}_{1 \mathrm{c}}$ and serum HDL-cholesterol results from a single laboratory were available for $\geq 98.8 \%$ of FDS patients compared with only 45.5 and $30.2 \%$, respectively, from multiple laboratories in ROSSO [4]) were both much greater in the FDS. The present analyses are therefore likely to have assessed the independent contribution of SMBG to diabetes-associated morbidity and mortality with greater validity. A healthy survivor effect may also have confounded the relationship between SMBG and non-fatal outcome in ROSSO, while these analyses were undertaken in FDS participants alive at the end of 5 years of follow-up.

After adjustment, SMBG was associated with an increased risk of cardiac death in patients not treated with insulin. Although the ROSSO investigators suggest that, based on apparent benefits, SMBG may be a surrogate for greater patient empowerment and physician interest, with consequently improved compliance [4], it is equally plausible that SMBG may represent a belated attempt to improve glycaemic control by mostly non-insulin-treated patients who become aware of symptoms of coronary insufficiency and heart failure. Retinopathy was the only non-fatal complication with which SMBG was independently associated, with a significantly lower relative risk in our 5-year cohort in both unadjusted and adjusted timedependent models. This could be a chance finding, but could also reflect the effect of other variables not measured in the FDS. It is not due to better glycaemic control in patients who perform SMBG, since SMBG use did not improve glycaemia [5]. The ROSSO study report does not contain a similar disaggregated analysis [4].

The results of the present study do not support a relationship between SMBG and improved survival in a well-characterised community-based sample of type 2 diabetic patients. We found evidence that SMBG was associated with an increased risk of cardiac death and a reduced risk of retinopathy. These conflicting results might reflect complex interactions between patient, physician and disease factors in particular circumstances, but may also represent the effects of confounding, incomplete covariate adjustment or chance.

Acknowledgements We thank FDS participants and staff. The Raine Foundation, University of Western Australia, funded the FDS.

Duality of interest We declare no current or past interest in relationship with a company/organisation that could benefit financially from the publication of our paper.

\section{References}

1. Gray A, Raikou M, McGuire A et al (2000) Cost effectiveness of an intensive blood glucose control policy in patients with type 2 
diabetes: economic analysis alongside randomised control trial (UKPDS 41). BMJ 320:1373-1378

2. Ipp E, Aquino RL, Christenson P (2005) Point: self-monitoring of blood glucose in type 2 diabetic patients not receiving insulin. The sanguine approach. Diabetes Care 28:1528-1530

3. Davidson MB (2005) Counterpoint: self-monitoring of blood glucose in type 2 diabetic patients not receiving insulin. A waste of money. Diabetes Care 28:1531-1533

4. Martin S, Schneider B, Heinemann L et al (2006) Self-monitoring of blood glucose in type 2 diabetes and long-term outcome: an epidemiological cohort study. Diabetologia 49:271-278

5. Davis WA, Bruce DG, Davis TME (2006) Is self-monitoring of blood glucose appropriate for all type 2 diabetic patients? The Fremantle Diabetes Study. Diabetes Care 29:1764-1770

6. Davis TM, Zimmet P, Davis WA, Bruce DG, Fida S, Mackay I (2000) Autoantibodies to glutamic acid decarboxylase in diabetic patients from a multiethnic Australian community: the Fremantle Diabetes Study. Diabet Med 17:667-674

7. Norman PE, Davis WA, Bruce DG, Davis TM (2006) Peripheral arterial disease and risk of cardiac death in type 2 diabetes: the Fremantle Diabetes Study. Diabetes Care 29:575-580

8. Holman CD, Bass AJ, Rouse IL, Hobbs MS (1999) Populationbased linkage of health records in Western Australia: development of a health services research linked database. Aust N Z J Public Health 23:453-459

9. Bradshaw PJ, Jamrozik K, Jelfs P, Le M (2000) Mobile Australians: a moving target for epidemiologists. Med J Aust 172:566, (Letter)

10. Davis WA, Norman PE, Bruce DG, Davis TM (2006) Predictors, consequences and costs of diabetes-related lower extremity amputation complicating type 2 diabetes: the Fremantle Diabetes Study. Diabetologia 49:2634-2641 